\title{
Biocalcarenitas como materiales de construcción en la iglesia de Santa Marina de Aguas Santas (Córdoba, España)
}

\section{Biocalcarenites as construction materials in Santa Marina de Aguas Santas Church at Cordoba, Spain}

\author{
J. Barrios Neira ${ }^{(*)}$, L. Montealegre(*), A. Ortega(*), J. E .Meroño(*) y M. J. Aguilera(*)
}

Recepción/Received: 30-VII-07

Aceptación/Accepted: 19-VI-08

Publicado online/Online publishing: 23-II-09

\section{RESUMEN}

Para la caracterización litológica y determinación del grado de alteración de los materiales pétreos se han empleado las siguientes técnicas: difracción de rayos $X$ (método del polvo), microscopía petrográfica (sobre lámina delgada) y microscopía de barrido con EDS (energía dispersiva de rayos $\mathrm{X}$ ), para determinar la composición química. El estado de degradación del material pétreo se ha cuantificado a partir del índice químico de alteración (CIW). Se han realizado cartografías sobre la fachada oeste: a) de las litologías presentes y b) de los diferentes tipos de alteración observados. La comparación de muestras del edificio con las de antiguas canteras ha permitido identificar la del Naranjo como la posible cantera de origen.

Palabras clave: calizas, restauración, alteraciones, microscopía óptica, difracción de rayos $\mathrm{X}$.
SUMMARY

This study consisted in characterizing the materials used to build Santa Marina de Aguas Santas Church at Cordoba and locating the original quarries. The techniques used in the lithological and chemical characterization included XRD, petrographic microscopy and electron dispersive scanning microscopy. The chemical index of weathering (CIW) was used to quantify the state of stone decay. The lithology and different types of alterations observed were mapped. A comparison of the material on the building to ancient quarries identified "Naranjo" as the possible site where the stone was originally quarried.

Keywords: limestone, restoration, alterations, optical microscopy, XRD.

\section{INTRODUCCIÓN}

En las últimas décadas el interés por la conservación del Patrimonio Histórico-Artístico ha tenido un gran auge. Es precisamente en este tiempo cuando el uso de combustibles fósiles y las emisiones de tipo industrial que aportan sustancias contaminantes a la atmósfera se han disparado, provocando daños espectaculares en los materiales de construcción, sobre todo en las áreas urbanas. Por este motivo, el protagonismo de los temas referentes a la restauración es cada día más creciente (1-3).

(*) Universidad de Córdoba. 
Uno de los objetivos de este trabajo es identificar los materiales de construcción empleados en los edificios pertenecientes al Patrimonio Histórico (en este caso, la iglesia de Santa Marina) y los tipos de alteraciones presentes, ya que es una labor importante previa a su restauración (4-5). Otro de ellos es la búsqueda para localizar las posibles canteras de donde fue extraído el material pétreo original (6-7). En toda restauración debe emplearse a ser posible el mismo material u otro de similares propiedades.

El estudio de las principales características de los diferentes templos fernandinos de la ciudad de Córdoba ha mostrado una gran semejanza en las características litológicas, mineralógicas y de otras propiedades (como el color) del material pétreo.

Esta iglesia se edificó en el último tercio del siglo XIII sobre un templo visigodo del siglo VII. Su construcción concluyó en el siglo XIV. Presenta una estética con influencias del gótico burgalés, del gótico cisterciense, con elementos del románico tardío y del mudéjar, que son precisamente los que contribuyen a restarle dureza y sobriedad (8). Está constituida por tres naves sin crucero y como en las restantes del grupo fernandino, la nave central es más alta que las laterales. La fachada principal está caracterizada por los potentes y asimétricos contrafuertes que marcan la separación de las naves y presenta rosetón, óculos y un tejaroz sobre las arquivoltas del arco ojival de entrada. Pertenece al grupo de los catorce templos construidos en la ciudad de Córdoba tras la conquista de la ciudad a los hispanomusulmanes en el siglo XIII, en tiempos de Fernando III el Santo. Es, junto con la iglesia de la Magdalena, una de las más antiguas de la capital y pueden considerarse como modelos de las otras iglesias fernandinas en Córdoba. Fue declarada Monumento Histórico-Artístico el 3 de junio de 1931.

La mayoría del material pétreo empleado es de la familia de las biocalcarenitas, de Edad Tortoniense, con cemento carbonatado micrítico y fósiles calizos (macrofauna de moluscos y microfauna de foraminíferos de escasa profundidad marina) muy abundantes y en buena parte fragmentados. En menor proporción hay sillares de una variedad de biomicrita arenosa de grano fino, y de biocalcarenita de carácter conglomerático (con clastos cuyo diámetro es superior a $2 \mathrm{~mm}$, y de naturaleza no carbonatada y con una abundancia próxima al $20 \%$ ) de la misma Edad Tortoniense. El conjunto se ha construido con materiales que corresponden a la denominada "Fácies de borde" de la Depresión Neógena del Guadalquivir (9).

\section{PARTE EXPERIMENTAL}

\subsection{Materiales}

Para poder determinar de qué cantera pudo ser extraído el material pétreo para la construcción, se han tomado muestras de los mismos litotipos de la iglesia y canteras (próximas a la ciudad) para su caracterización mineralógica y petrológica.

La Figura 1 muestra la geografía y geología de la zona, así como la ubicación y estratigrafía de algunas de las canteras antiguas y actuales próximas a Córdoba.

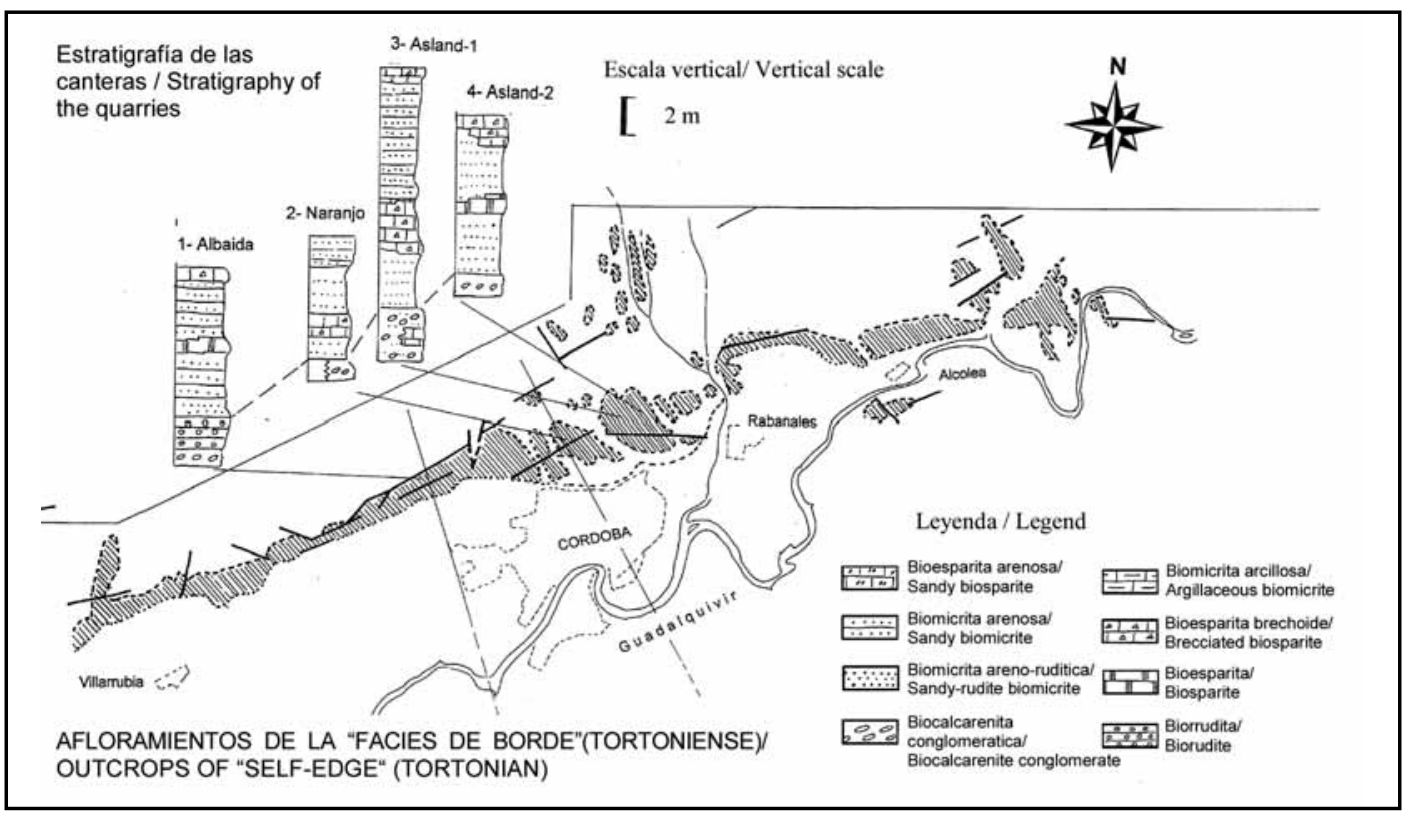

Figura 1. Estratigrafía de las canteras. 
Se ha realizado un muestreo de material pétreo en sillares alterados y sin alterar de la fachada oeste de la iglesia cuya ubicación se indica en las Figuras 2, 3 y 4 . Los números indicados en las muestras de las tablas I y II corresponden a una serie de muestras de la fachada
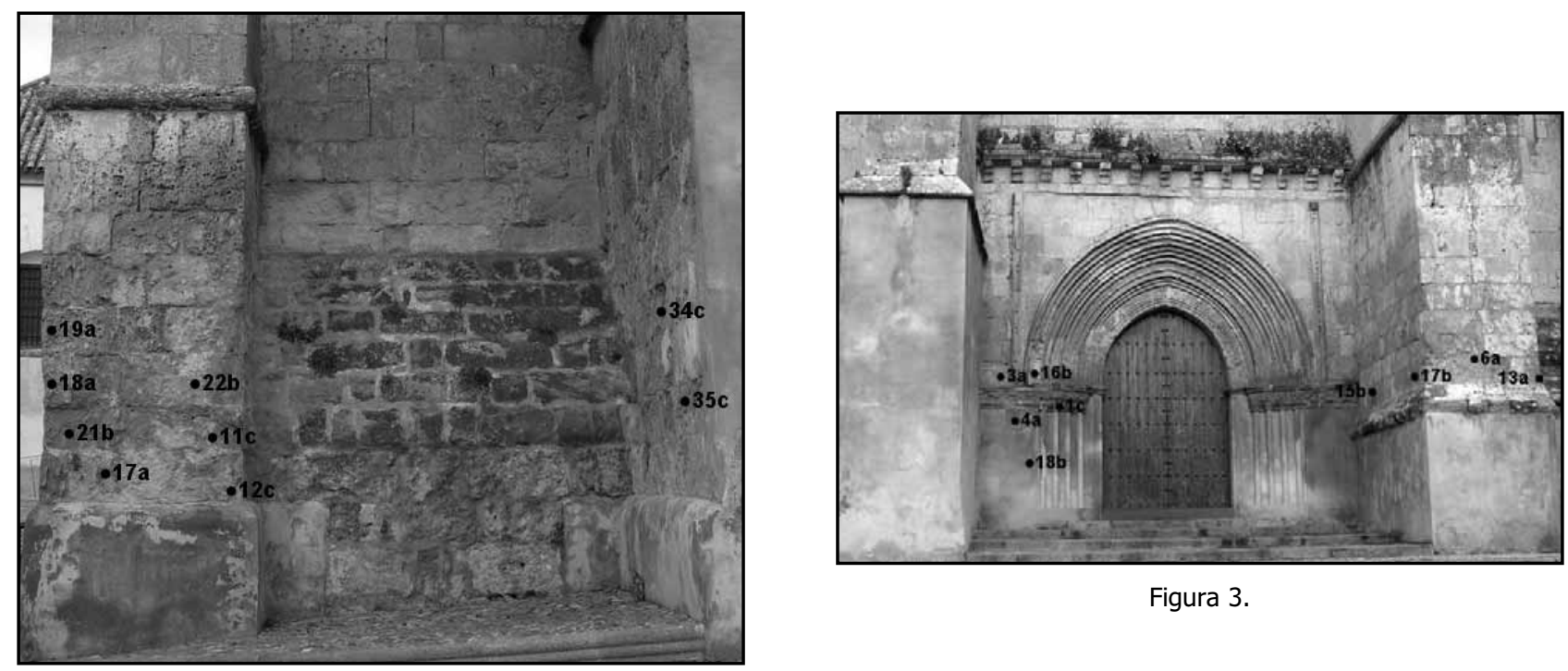

Figura 3.

Figura 2 de cada pastilla son los que pueden verse en las Tablas II (edificio) y IV (canteras).

El estudio petrográfico en lámina delgada se realizó sobre fragmentos de mayor tamaño.

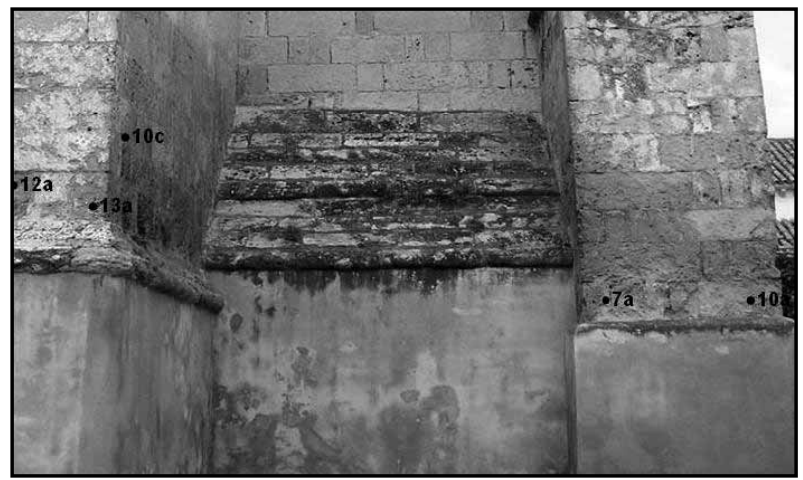

Figura 4.

Figuras 2, 3 y 4 . Fachada oeste de la iglesia de Santa Marina.

oeste de la iglesia. Cada valor expresado en el casillero "muestra" (en las tablas) es un valor representativo y no puntual. En las canteras el muestreo se ha realizado sobre zonas expuestas y no expuestas a la intemperie (en la actualidad).

Para el análisis por difracción de rayos $\mathrm{X}$ y el microanálisis EDS, las muestras (edificio y canteras) se trituraron en mortero de ágata hasta pulverización y posteriormente se compactaron en pastillas de $13 \mathrm{~mm}$ de diámetro. Sobre cada pastilla se efectuaron seis medidas (microanálisis EDS) y los valores medios de los datos obtenidos
Para identificar la posible cantera de origen, se han comparado los datos del material del edificio con los de cuatro canteras históricas (Albaida, Asland, Naranjo y Patriarca), por la mayor semejanza de las características de sus materiales con las de los empleados en el templo.

Se ha empleado el índice CIW (10) para poder realizar una cuantificación del estado de alteración [1]:

$$
\mathrm{CIW}=\left[\frac{\mathrm{Al}_{2} \mathrm{O}_{3}}{\mathrm{Al}_{2} \mathrm{O}_{3}+\mathrm{CaO}+\mathrm{Na}_{2} \mathrm{O}}\right] \times 100
$$




\subsection{Técnicas}

En el estudio de las muestras del edificio y canteras se han aplicado las siguientes técnicas de análisis:

Difractómetro de Rayos X Siemens $D 5000$ con radiación monocromática Co $\mathrm{K} \alpha(\lambda=0.179 \mathrm{~nm} ; 40 \mathrm{KV}, 25 \mathrm{~mA})$ para determinar la composición mineralógica. La posición inicial del goniómetro del difractómetro se fijó en $2 \theta=2^{\circ}$ y la posición final en $2 \theta=40^{\circ}$, con un paso de 0.05 y una velocidad de paso de 5 segundos. Para la determinación semicuantitativa (\%) de las diferentes especies presentes en las muestras se han utilizado los poderes reflectantes correspondientes (9). A partir de los difractogramas de rayos $X$ (método del polvo) se calculó el área del pico de mayor intensidad de cada especie presente dividida por su poder reflectante $\left(\mathrm{A}_{1} / \mathrm{Pf}_{1}, \mathrm{~A}_{2} / \mathrm{Pf}_{2} \ldots\right)$ y dividido todo ello por la suma de los valores calculados para todas las especies presentes, según la ecuación [2]:

$$
(\%)_{i}=\frac{A_{i} / P f_{i}}{\Sigma\left[A_{j} / P f_{j}\right]}
$$

Fotomicroscopío petrográfico Carl Zeiss II POL, equipado con cámara fotográfica y luz transmitida. Mediante lámina delgada se puede determinar la mineralogía, textura, petrografía, edad (mediante los microfósiles presentes), facies y ambiente sedimentario.

Microscopio electrónico de barrido JEOL JSM 6300 equipado con sistema de microanálisis EDS para determinar la composición química del material pétreo.

\section{RESULTADOS Y DISCUSIÓN}

\subsection{Edificio}

El estudio del material pétreo del edificio indica que al igual que en la mayoría de los monumentos cordobeses, los sillares son biocalcarenitas de Edad Tortoniense y facies marina. En la toma de muestras de los sillares en el edificio se tuvieron en cuenta: los distintos litotipos, la diferente ubicación y el distinto grado de alteración (Figuras 2, 3 y 4).

La Tabla I muestra los datos registrados mediante difracción de rayos $\mathrm{X}$. Se dan los valores medios de composición mineralógica (\%) de las muestras tomadas en la iglesia.

Tabla I

Composición mineralógica (\%). Fachada oeste de Santa Marina.

\begin{tabular}{|c|c|c|c|c|c|}
\hline Muestra & Yeso & Cuarzo & Calcita & Feldespato & Arcilla \\
\hline $2 a$ & 10 & 20 & 70 & - & - \\
\hline $3 a$ & 12 & 18 & 70 & - & - \\
\hline $4 a$ & - & 20 & 80 & Trazas & - \\
\hline $6 a$ & 17 & 3 & 80 & - & Trazas \\
\hline $7^{a}$ & - & 5 & 95 & - & Trazas \\
\hline $10 a$ & Trazas & 24 & 76 & Trazas & Trazas \\
\hline $12 \mathrm{a}$ & - & 15 & 85 & Trazas & Trazas \\
\hline $13 a$ & - & 25 & 75 & - & Trazas \\
\hline $14 a$ & - & 15 & 60 & 25 & Trazas \\
\hline $17 a$ & Trazas & 80 & Trazas & 20 & - \\
\hline $18 a$ & - & 10 & 90 & - & Trazas \\
\hline $19 a$ & 5 & 5 & 90 & Trazas & - \\
\hline $15 \mathrm{~b}$ & - & 5 & 90 & 5 & Trazas \\
\hline $16 \mathrm{~b}$ & - & 12 & 88 & - & - \\
\hline $17 \mathrm{~b}$ & - & Trazas & 100 & Trazas & Trazas \\
\hline $18 \mathrm{~b}$ & - & 35 & 65 & Trazas & - \\
\hline $21 b$ & Trazas & Trazas & 100 & - & - \\
\hline $22 b$ & 3 & 7 & 90 & Trazas & - \\
\hline $10 \mathrm{c}$ & - & 25.0 & 75.0 & - & Trazas \\
\hline $11 \mathrm{c}$ & 7.0 & 30.0 & 63.0 & Trazas & - \\
\hline $12 \mathrm{c}$ & - & 20.0 & 70.0 & 10.0 & - \\
\hline $31 \mathrm{c}$ & - & 43.0 & 56.0 & - & - \\
\hline $34 c$ & 31.0 & 14.0 & 51.0 & 4.0 & Trazas \\
\hline $35 c$ & - & Trazas & 100.0 & Trazas & Traza \\
\hline
\end{tabular}


En la Tabla II puede verse la composición química (\%) de las muestras tomadas en el edificio. Los datos de composición química se han determinado mediante el EDS para cada una de las muestras de material pétreo, tanto de la iglesia como de canteras. predominio de biocalcarenita con textura clástica, arenosa y de color amarillento. Solamente de forma local son visibles las calcarenitas de facies conglomeráticas. Existen también diversos materiales artificiales, como es el ladrillo situado en algunas cornisas. La

Tabla II

Composición química (\%) y CIW. Fachada oeste de Santa Marina.

\begin{tabular}{|c|c|c|c|c|c|c|c|c|c|c|}
\hline Muestra & $\mathbf{C O}_{\mathbf{2}}$ & $\mathbf{N a}_{\mathbf{2}} \mathbf{O}$ & $\mathbf{M g O}$ & $\mathbf{A l}_{\mathbf{2}} \mathbf{O}_{\mathbf{3}}$ & $\mathbf{S i O 2}$ & $\mathbf{S O 3}$ & $\mathbf{K}_{\mathbf{2}} \mathbf{O}$ & $\mathbf{C a O}$ & $\mathbf{F e}_{\mathbf{2}} \mathbf{O}_{\mathbf{3}}$ & $\mathbf{C I W}^{\mathbf{2}}$ \\
\hline $2^{\mathrm{a}}$ & 44.9 & - & - & 0.8 & 9.1 & 6.6 & - & 36.6 & 2.1 & 2.1 \\
\hline $3^{\mathrm{a}}$ & 21.2 & - & 0.2 & 1.1 & 2.0 & 19.2 & 0.4 & 54.5 & 1.4 & 2.0 \\
\hline $4^{\mathrm{a}}$ & 15.8 & 2.0 & 1.6 & 2.5 & 3.5 & 1.1 & 1.7 & 67.0 & 4.8 & 3.5 \\
\hline $6^{\mathrm{a}}$ & 43.8 & - & 1.4 & 1.2 & 17.6 & 5.5 & 0.4 & 29.0 & 1.1 & 4.0 \\
\hline $7^{\mathrm{a}}$ & 55.8 & 0.8 & 0.7 & 1.8 & 5.6 & 1.5 & 0.6 & 30.7 & 2.5 & 5.4 \\
\hline $10^{\mathrm{a}}$ & 47.6 & 0.8 & 0.9 & 2.2 & 10.0 & 1.4 & 1.6 & 31.0 & 4.5 & 6.5 \\
\hline $12^{\mathrm{a}}$ & 51.5 & 0.6 & 1.5 & 2.4 & 9.1 & 1.0 & 1.1 & 30.1 & 2.7 & 7.3 \\
\hline $13^{\mathrm{a}}$ & 51.0 & 0.7 & 0.0 & 2.5 & 15.2 & 0.8 & 0.8 & 27.3 & 1.7 & 8.2 \\
\hline $14^{\mathrm{a}}$ & 42.7 & 3.4 & 5.0 & 8.3 & 21.5 & 0.3 & 0.7 & 11.6 & 6.5 & 35.6 \\
\hline $18^{\mathrm{a}}$ & 33.7 & 1.6 & 1.3 & 3.1 & 11.1 & - & 1.1 & 44.8 & 3.3 & 6.3 \\
\hline $19^{\mathrm{a}}$ & 47.1 & 0.2 & 0.6 & 0.8 & 4.1 & 2.3 & 1.1 & 41.4 & 2.4 & 2.1 \\
\hline $15 \mathrm{~b}$ & 48.7 & 1.3 & 0.9 & 2 & 7.3 & 0 & 1 & 36 & 2.8 & 5.1 \\
\hline $16 \mathrm{~b}$ & 37.5 & 2.1 & 0.5 & 2.4 & 8.5 & 0.2 & 1.2 & 46.2 & 1.4 & 4.7 \\
\hline $17 \mathrm{~b}$ & 57.8 & 1 & 0.4 & 1.6 & 4.5 & 0 & 0.5 & 32.5 & 1.7 & 4.6 \\
\hline $18 \mathrm{~b}$ & 17 & 6.7 & 2.6 & 5 & 12.8 & 1.3 & 2.5 & 46 & 6.3 & 8.7 \\
\hline $21 \mathrm{~b}$ & 51 & 0.5 & 1.3 & 2 & 7.8 & 0.6 & 1.1 & 33.9 & 1.8 & 5.5 \\
\hline $22 \mathrm{~b}$ & 49 & 0.2 & 0.8 & 1.4 & 4.9 & 0.9 & 0.6 & 40.3 & 1.6 & 3.3 \\
\hline $10 \mathrm{c}$ & 53.7 & 0.5 & 1.1 & 1.4 & 8.4 & 7.5 & 0.6 & 25.4 & 1.4 & 5.1 \\
\hline $11 \mathrm{c}$ & 50.4 & 1.2 & 0.7 & 2.0 & 22.0 & 0.2 & 2.1 & 20.5 & 0.9 & 8.4 \\
\hline $12 \mathrm{c}$ & 49.3 & 0.3 & 0.1 & 2.3 & 25.1 & 0.5 & 0.7 & 20.7 & 1.0 & 9.9 \\
\hline $31 \mathrm{c}$ & 41.5 & 2.0 & 1.5 & 4.2 & 19.8 & 0.5 & 2.1 & 24.9 & 3.5 & 13.5 \\
\hline $34 \mathrm{c}$ & 47.9 & 0.6 & 1.6 & 3.1 & 11.9 & 14.5 & 1.1 & 16.5 & 2.8 & 15.3 \\
\hline $35 \mathrm{c}$ & 61.5 & 0.9 & 0.8 & 0.7 & 3.4 & - & 0.7 & 31.1 & 1.0 & 2.1 \\
\hline & & & & & & & & & & \\
\hline & & & & & & & \\
\hline & & & & & & & & & \\
\hline
\end{tabular}

La presencia de yeso puede deberse a contaminación antrópica, ya que dicho material no se encuentra en las canteras.

Para poder cuantificar el estado de alteración de los materiales estudiados en la iglesia se ha calculado el índice CIW (10). A partir de los datos de composición química obtenidos (Tabla II), se obtiene un valor del CIW, que permite la semicuantificación del estado de degradación. El CIW da un valor igual a 2 en muestras sin degradar y 20 para muestras degradadas. Las muestras degradadas dan valores más altos del CIW, como era de esperar y coinciden con las muestras que presentan un alto grado de degradación en el edificio.

En la Figura 5 se muestra la cartografía litológica de la puerta oeste de la iglesia, donde puede verse el

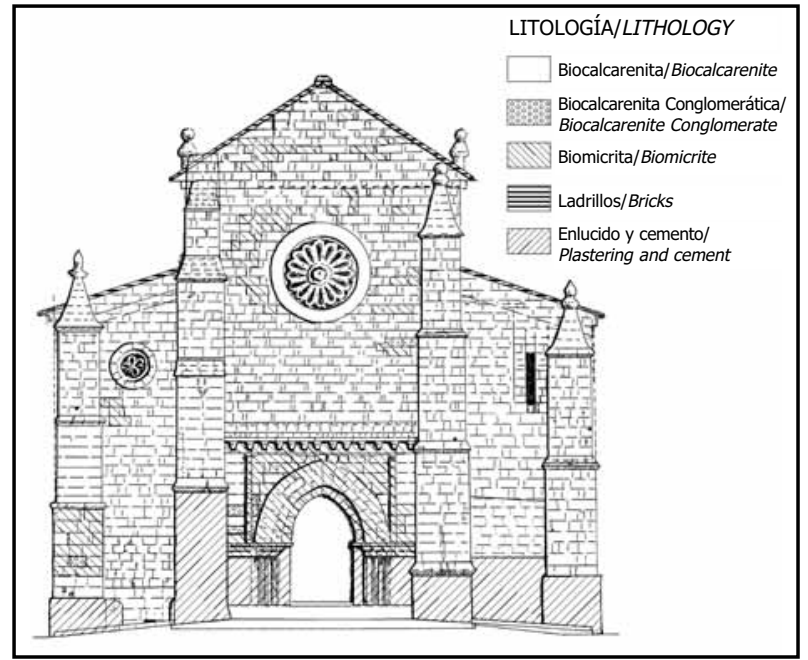

Figura 5. Cartografía litológica de la fachada oeste de Santa Marina. 
mayor parte de las biomicritas arenosas están situadas en las partes bajas del edificio.

El aspecto macroscópico de los distintos litotipos de biocalcarenitas presentes en la fachada oeste pueden verse en las Figuras: 6 (biomicrita), 7 (biomicrita arenosa), 8 (biomicrita conglomerática) y 9 (bioesparita).

Mediante microscopía se han clasificado las biocalcarenitas, a partir de las relaciones: clastos / matriz, fósiles / matriz y textura según los modelos de clasificación de rocas sedimentarias siendo las más representativas las biomicritas (según Folk) y "pakestone" (según Dunham).

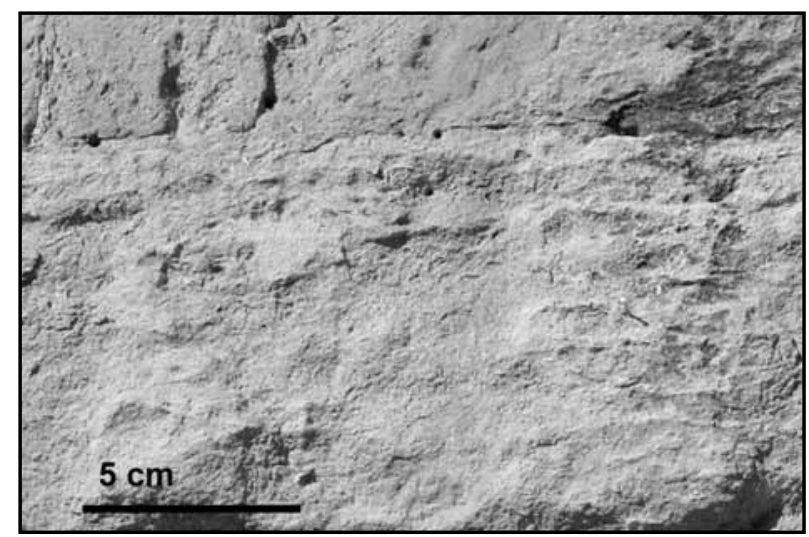

Figura 6. Biomicrita (macro) .

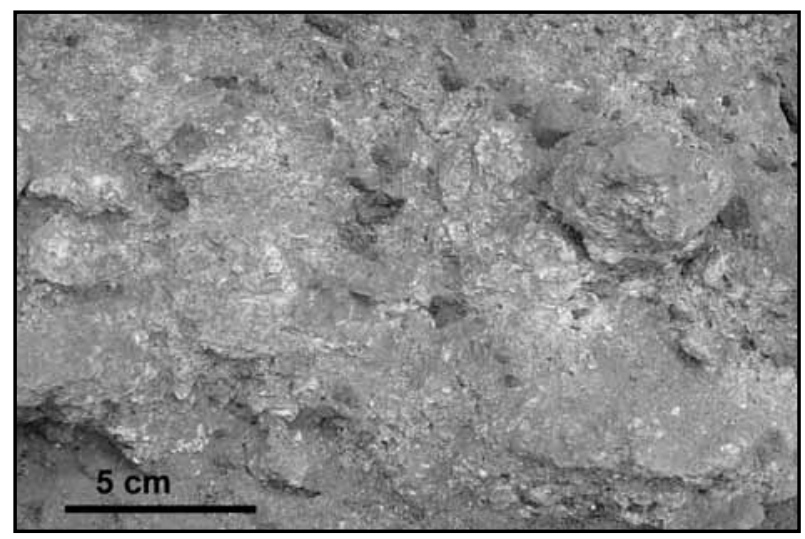

Figura 8. Biomicrita conglomerática.

En algunos casos las biocalcarenitas son biomicritas arenosas por la abundancia en cuarzo que presentan y por su textura marcadamente detrítica también son "pakestone" (Dunham). En las biomicritas conglomeráticas (poco frecuentes en el edificio) hay una gran abundancia de clastos rudíticos (no carbonatados) de diámetro superior a $2 \mathrm{~mm}$ (apreciables a simple vista). En general presentan una clastificación o fragmentación bastante acentuada de la fauna. Las bioesparitas son semejantes a las biomicritas, pero ofrecen menos porcentaje en granos detríticos, y el cemento es más cristalino. Se designan como "grainstone" (Dunham). Son sin embargo muy escasas en el edificio.

Las Figuras 10 y 11 muestran el aspecto en lámina delgada de las biomicritas y biomicritas arenosas de los sillares del edificio. En la Figura 10 puede verse una matriz fina con restos rotos de microfauna, foraminíferos, arenaceos (arriba izquierda), películas de oxihidróxidos de hierro y en la Figura 11 también puede verse matriz fina con restos rotos de microfauna, foraminíferos y abundantes cuarzos (blanco).

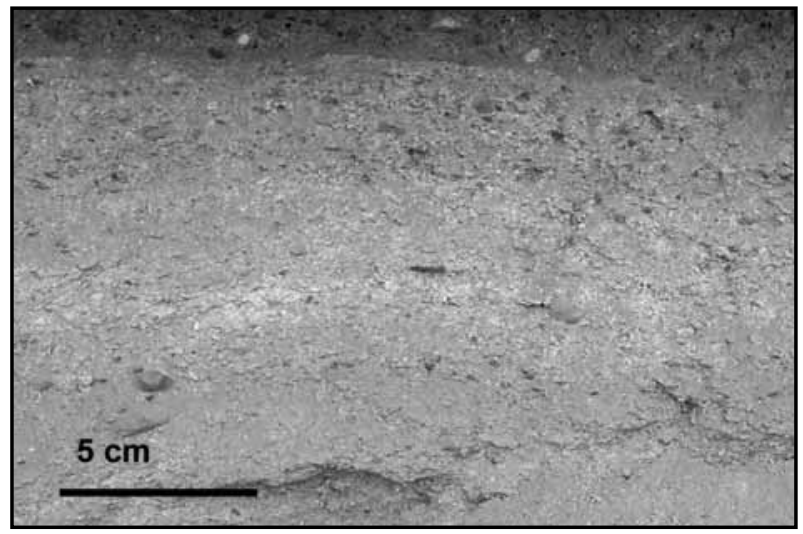

Figura 7. Biomicrita arenosa.

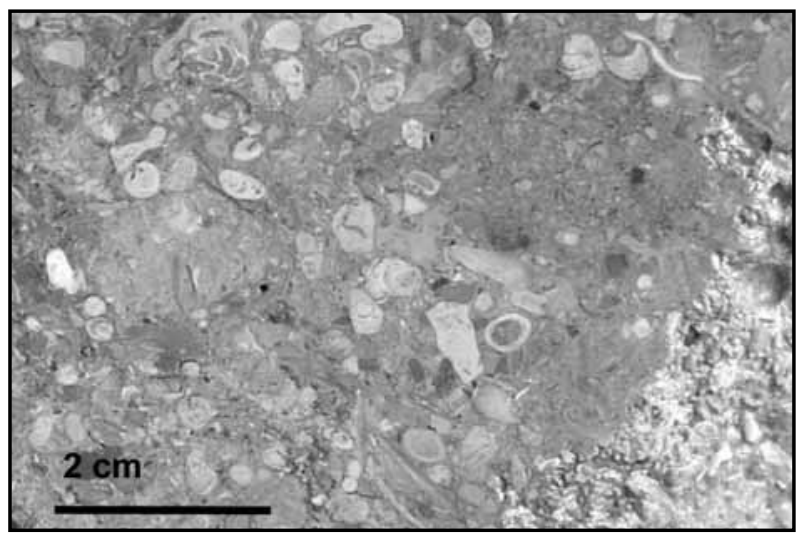

Figura 9. Bioesparita.

La microestructura de los sillares de biomicrita (el material más abundante) en la iglesia y en la cantera del Naranjo es muy semejante en: diámetro y forma de las partículas, tanto detríticas (cuarzo, fragmentos de roca, etc.) como de la matriz cabonatada, clastificación (fragmentación) y conservación de la fauna. 
La mineralogía detectada en lámina delgada es semejante a la observada por difracción de rayos X: calcita acompañada de cuarzo con escasos feldespatos, arcillas y ocasionales oxihidróxidos de hierro. En lámina delgada entre los microfósiles pueden identificarse globigerínidos, heterosteginas, nodosáridos, arenáceos y otros, y entre la macrofauna (bastante clastificada) abundan los restos de algas calcáreas, briozoarios, lamelibranquios, y sedas y placas de erizos.

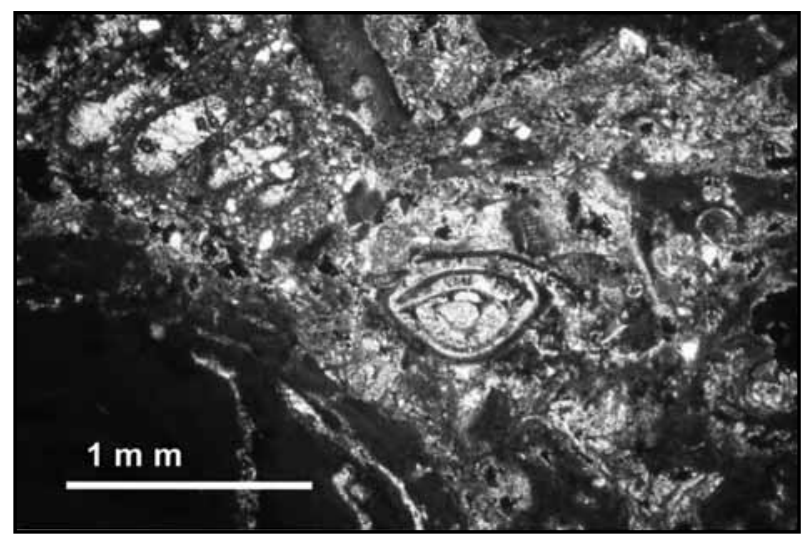

Figura 10. Biomicrita.

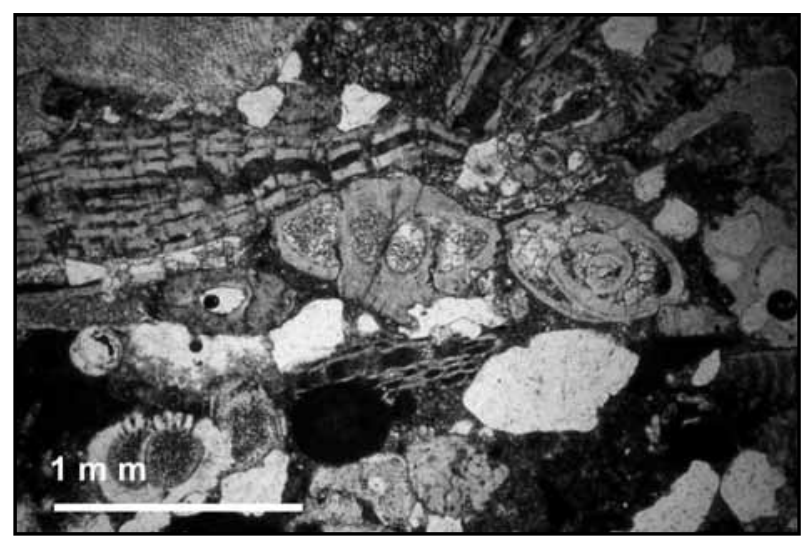

Figura 12. Biomicrita.

Las Figuras 12 y 13 (lámina delgada) son de los mismos tipos de materiales. En la Figura 12 (biomicrita) puede verse matriz oscura micrítica con foraminíferos y cuarzo. En la Figura 13 (biomicrita arenosa) se observa una pasta micrítica, placas de fósiles con canales rellenos de oxihidróxidos de hierro, arcillas rojizas en huecos, abundantes clastos de cuarzo y opacos.

\subsection{Canteras}

En las Tablas III y IV se dan valores medios mineralógicos y químicos de varias canteras. Para determinar la composición química y mineralógica del material de la cantera del Naranjo, se tomaron varias muestras: en profundidad (muestras no degradadas) e igualmente en superficie (muestras degradadas).

Se han determinado sobre materiales pétreos de las cuatro canteras diferentes propiedades como: resistencia a la compresión $\left(\mathrm{Nw} / \mathrm{m}^{2}\right)(\mathrm{I})$, velocidad de

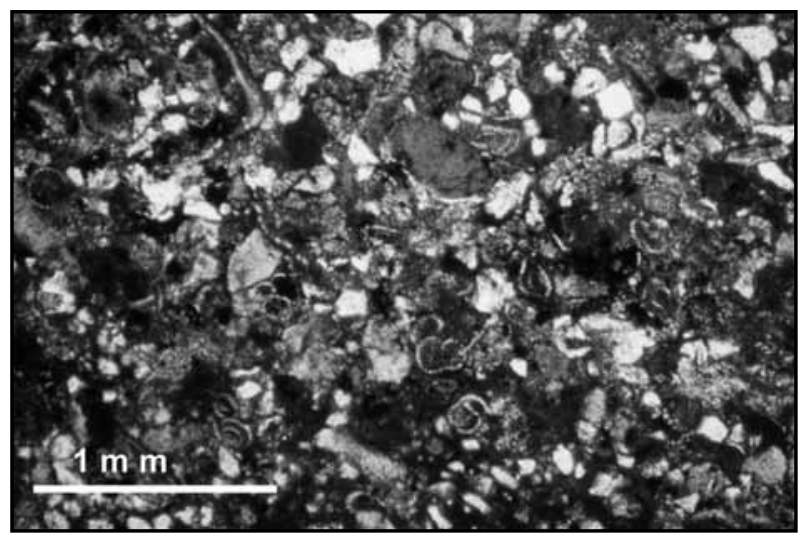

Figura 11. Biomicrita arenosa.

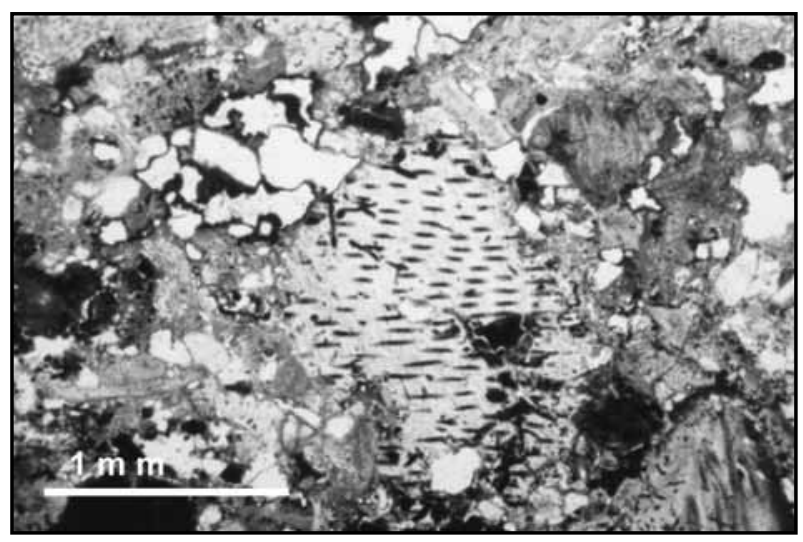

Figura 13. Biomicrita arenosa.

ultrasonido (km/s) (II), porcentaje de porosidad (III) y de fósiles(IV).
a) Albaida
(I) $190-150$
(II) 2.9
(III) $<15$
(IV) $40-50$
b) Patriarca
80-60
3.1
15
$0-15$
$15-20$
c) Asland
$80-50$
d) Naranjo
$180-140$
3.0
10-15
20-30
$30-40$

La cantera de la Albaida es similar en color, compactación y grado de conservación de la fauna (entre otras características) con los sillares cartografiados como biocalcarenitas en la iglesia de Santa Marina, pero la 
resedimentación es menos patente. El diámetro de partícula y la abundancia de macrofauna es mayor en esta cantera que en la del Naranjo.

La del Patriarca muestra abundancia de facies conglomeráticas y no ofrece volumen significativo de extracción, por lo que parece estaba prácticamente agotada en la época de construcción de las iglesias fernandinas.

En la de Asland se observa abundancia de secuencias esparíticas compactas (escasas en Santa Marina) y biomicritas de color gris (inexistentes en dicho edificio).

La del Naranjo presenta las dimensiones suficientes de los niveles extraídos de biomicritas y biomicritas arenosas, materiales que se encuentran en la iglesia con mayor abundancia. Al analizar la apariencia del material en este afloramiento se ha visto que es algo arenosa, pero presenta una buena homogeneidad. En la parte de la cantera más asequible afloran los niveles más altos de la formación de borde, que son los más arenosos y tableados, presentando además ciertos contenidos de arcillas. Estos materiales coinciden bastante bien con los sillares de la puerta y zonas bajas del edificio, precisamente las cartografiadas como biomicrita arenosa. Los niveles más bajos de la cantera muestran un mayor margen de variabilidad, pero se asemejan a buena parte de los sillares cartografiados como biocalcarenitas en general. Entre las características del material estudiado están: un carácter más o menos masivo de los estratos aflorantes cortados durante la extracción. Las rocas aflorantes actualmente en la cantera son en general biocalcarenitas, de tamaño de grano medio con abundante microfauna (fragmentada), con bajos contenidos rudíticos y un porcentaje visible de oxihidróxidos de hierro, lo que le confiere un color amarillo más saturado que en otros yacimientos.

La comparación entre los datos analizados (mineralógicos, petrológicos, mecánicos y de color) de la antigua cantera del Naranjo con los de los sillares de la iglesia y con datos conocidos de otros afloramientos próximos de esta formación marina del Tortoniense, apoyan una mayor probabilidad de que se utilizase esta cantera como origen de los materiales empleados en la construcción de la iglesia de Santa Marina. Es significativa la gran semejanza en la microfauna fósil de sillares (edificio) y cantera del Naranjo.

Tabla III

Composición mineralógica (\%). Material de canteras.

\begin{tabular}{|l|c|c|c|c|}
\hline \multirow{2}{*}{ Canteras } & \multicolumn{3}{c|}{ Composición mineralógica } \\
\cline { 2 - 5 } & Cuarzo & Calcita & Feldespato & Arcillas \\
\hline Albaida & $23-28$ & $40-55$ & $7-12$ & $4-8$ \\
\hline Posadas & $10-15$ & $70-82$ & $3-5$ & $2-7$ \\
\hline Asland & $5-15$ & $65-80$ & $2-5$ & $1-3$ \\
\hline Naranjo & $10-25$ & $65-85$ & $3-6$ & 2 \\
\hline No degradado (Naranjo) & 15 & 80 & 3 & 2 \\
\hline Degradado (Naranjo) & 20 & 75 & 3 & \\
\hline
\end{tabular}

Tabla IV

Composición química (\%). Material de canteras.

\begin{tabular}{|l|c|c|c|c|c|c|c|c|}
\hline \multicolumn{1}{|c|}{ Canteras/Quarries } & $\mathbf{C O}_{\mathbf{2}}$ & $\mathbf{C a O}$ & $\mathbf{A l}_{\mathbf{2}} \mathbf{O}_{\mathbf{3}}$ & $\mathbf{F e}_{\mathbf{2}} \mathbf{O}_{\mathbf{3}}$ & $\mathbf{K}_{\mathbf{2}} \mathbf{0}$ & $\mathbf{M g O}$ & $\mathbf{S i O}_{\mathbf{2}}$ & $\mathbf{S O}_{\mathbf{3}}$ \\
\hline Albaida & 49 & 25.2 & 1.6 & 2.11 & 1.16 & 1.04 & 19.85 & 0.05 \\
\hline Posadas & 45.75 & 37.9 & 1.65 & 1.04 & 0.63 & 0.43 & 12.57 & 0 \\
\hline Asland & 49.25 & 36.55 & 1.31 & 0.94 & 0.49 & 0.38 & 11.08 & 0 \\
\hline Naranjo & 45.5 & 39.3 & 0.81 & 1.1 & 0.4 & 0.51 & 12.35 & 0 \\
\hline No degradado (Naranjo) & 45.5 & 39.3 & 0.81 & 1.1 & 0.4 & 0.51 & 12.35 & 0 \\
\hline Degradado (Naranjo) & 28 & 22.4 & 7.3 & 8.5 & 1.9 & 1.8 & 29.4 & 0 \\
\hline
\end{tabular}




\subsection{Alteraciones}

Para mostrar la ubicación de los distintos tipos de alteraciones presentes en la fachada oeste de la iglesia se han realizado varias cartografías (Figuras. 14 y 15).

La Figura 14 permite ver la localización de alteraciones generadas por agentes abióticos como son: picado, alveolización, alteración diferencial, arenización, fisuras y pátinas oscuras.

Existen fisuras y grietas sobre todo en las zonas de unión de los contrafuertes con las fachadas. En el panel oeste puede verse una importante fisura que va de arriba hacia abajo en toda la fachada.

Las fisuras observadas no corresponden a procesos de compactación diferencial del suelo y su análisis geométrico evidencia movimientos horizontales orientados en la misma dirección (o en paralelo) respecto a los mecanismos tensoriales que se observan en edificios de Carmona (Sevilla), Córdoba, etc., provocados por el terremoto de Lisboa (1 de noviembre de 1755) que fue uno de los más destructivos (alto grado en la escala Richter) en la historia de la Península Ibérica. Estos mecanismos de rotura coinciden en su alineación con la famosa falla del Guadalquivir (de cuya actividad existen referencias desde la antigüedad), que es un ramal de la gran fractura transformante que se prolonga desde el Atlántico.

La arenización es una de las alteraciones más abundantes especialmente en la zona inferior del edificio. El picado y la alveolización se encuentran en mayores extensiones y con cierto grado de intensidad en el contrafuerte más septentrional.

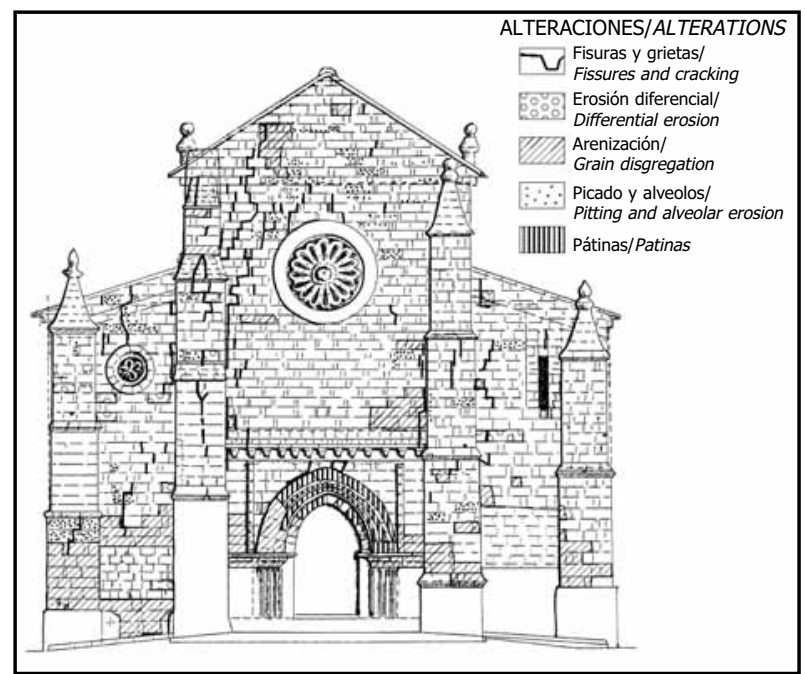

Figura 14. Cartografía de las alteraciones originadas por agentes abióticos (Santa Marina).
Las pátinas aparecen sobre todo en las partes internas de arcadas y rosetones.

La Figura 15 muestra las alteraciones debidas a los agentes bióticos (algas, musgos, líquenes y plantas superiores) (11). Las fachadas $\mathrm{N}$ y $\mathrm{W}$ del edificio son las más afectadas por la lluvia (12).

Las más abundantes son las algas, sobre todo en las partes inclinadas de los contrafuertes (y tejados) y en las zonas situadas debajo de éstos. Los musgos están en las cornisas y especialmente en las zonas inclinadas y salientes. En estas zonas puede verse la presencia de plantas superiores (Figura 3). Los líquenes se hallan en las partes altas de los contrafuertes y en la parte saliente sobre la arcada de la puerta.

\section{CONCLUSIONES}

Santa Marina de Aguas Santas fue construida con materiales extraídos de canteras de biocalcarenitas del Tortoniense, situadas en las cercanías de Córdoba.

El empleo del material del Naranjo puede apoyarse también en la similitud de características físicas y microestructurales de los materiales.

La presencia de yeso puede deberse a una posible contaminación antrópica.

La cartografía litológica permite señalar que el material dominante son las biomicritas y en menor cuantía las biomicritas arenosas de grano más fino. Sólo un pequeño porcentaje corresponde a bioesparitas y biomicritas

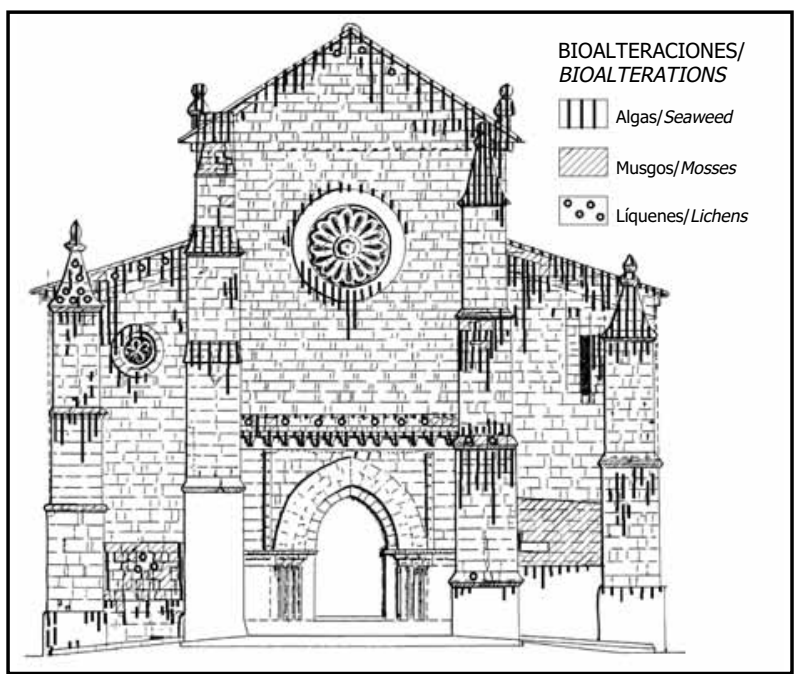

Figura 15. Cartografía de las alteraciones originadas por agentes bióticos (Santa Marina). 
conglomeráticas, niveles que también forman parte de las facies de borde del Tortoniense.

La comparación entre los datos (mineralógicos y químicos) obtenidos de muestras tomadas en la iglesia y en las canteras, ha facilitado la selección de la del Naranjo como la cantera de la cual pudo extraerse el material empleado en la construcción inicial de la iglesia.

Los valores de CIW calculados indican que el grado de alteración del material en esta fachada no es muy alto.
Un aumento del grado de degradación parece ir unido en general a la disminución del contenido en calcio y un aumento en el \% de sodio y potasio.

El terremoto de Lisboa parece ser el causante de la mayor parte de las fisuras.

La bioalteración (algas) está generalizada en zonas de derrame o retención de agua.

\section{REFERENCIAS}

(1) Alcalde, M.; Villegas, R.: "Indicadores de alteración de los materiales pétreos. Metodología de diagnóstico y evaluación de tratamientos para la conservación de los edificios históricos", Editorial Comares, Junta de Andalucía (2003), cap. 2., pp. 58-71.

(2) Esbert, R.; Marcos, R.: "La deterioración de las piedras de la Catedral de Oviedo. 2a parte: formas y fenómenos de alteración". Mater. Construcc., 186 (1982), pp. 79-88.

(3) Sebastián, E.: "La difracción de rayos X en la conservación del patrimonio arquitectónico. Metodología de diagnóstico y evaluación de tratamientos para la conservación de los edificios históricos", Editorial Comares, Junta de Andalucía (2003), Cap. 3, pp. 96-103.

(4) Barrios, J.; Montealegre, L.; Navas, J.; Nieto, M.: "Characteristics and types of alteration in materials found in the west façade of the Mosque of Córdoba (Spain)". Proceeding III International Symposium on the Conservation of Monuments in Mediterranean Basin, Venecia (1994), pp. 755-761.

(5) Barrios, J.; Montealegre, L.; Nieto, M.: "El Alcázar de los Reyes Cristianos, materiales pétreos y canteras", Proceedings III International Congress of Restoration of Buildings and Architectural Heritage, Granada (1996), pp. 238-242.

(6) Montealegre, L.: "La Geología Cordobesa. Córdoba Capital", Caja Provincial de Ahorros de Córdoba, Córdoba (1994), V. 3, pp. 14-24.

(7) Fort, R.: "Localización de antiguas canteras utilizadas en el patrimonio monumental. Degradación y Conservación de Patrimonio Arquitectónico", Editorial Complutense, Madrid (1996), cap. 5, pp. 310-316.

(8) Pérez, M.: "Estudio histórico-artístico de la Iglesia Parroquial de Santa Marina de Aguas Santas de Córdoba", Publicaciones de la Universidad de Córdoba y Obra Social y Cultural Cajasur, Córdoba (1998).

(9) Montealegre, L.: "Estudio mineralógico en sedimentos y suelos de la Depresión del Guadalquivir", Tesis Doctoral, Universidad de Granada (1976).

(10) Harnois, L.: "The CIW index: A new chemical index of weathering", Sedimentary Geology, 55 (3-4) (1988), pp. 319-322. doi:10.1016/0037-0738(88)90137-6

(11) Warscheid, T.; Braams, J. "Biodeterioration of a stone: a review", International Biodeterioration \& Biodegradation, 6 (4). (2000), pp. 343-368. doi:10.1016/S0964-8305(00)00109-8

(12) Domínguez, P.: "Rasgos climáticos del término municipal Córdoba capital", Caja Provincial de Ahorros de Córdoba (1994), V.3, pp. 25-30. 